SHS Web of Conferences 21, 01002 (2015)

DOI: $10.1051 /$ shsconf/ 20152101002

(C) Owned by the authors, published by EDP Sciences, 2015

\title{
Épistémologie de la biologie et conceptualisation du vivant chez des futurs enseignants et biologistes
}

\author{
Catherine Simard ${ }^{1, \text { a }}$, Léon Harvey ${ }^{1}$ et Ghislain Samson ${ }^{2}$ \\ ${ }^{1}$ Université du Québec à Rimouski, 300, allée des Ursulines, C. P. 3300, succ. A, Rimouski, Québec, G5L 3A1, \\ Canada \\ ${ }^{2}$ Université du Québec à Trois-Rivières, 3351 Boulevard des Forges, Trois-Rivières, Québec, G9A 5H7, Canada
}

\begin{abstract}
Résumé. L'ensemble des résultats présentés dans cet article relève d'une étude doctorale qui s'est intéressée à l'histoire de la biologie et aux grandes conceptions qui ont alimenté la construction des savoirs en biologie jusqu'à présent (Simard, 2015 [1]). À cet égard, un ensemble de conceptions du vivant a été sélectionné et circonscrit afin de développer un nouvel outil d'évaluation estimant la présence de l'une ou l'autre de ces conceptions chez les futurs enseignants et biologistes. Pour certaines conceptions s'avérant désuètes, la problématique est qu'elles se voient reconduites, encore aujourd'hui, dans la pensée scientifique. C'est alors en termes d'obstacle épistémologique historique à une conceptualisation contemporaine du vivant qu'elles sont appréhendées. Cette caractérisation de la conceptualisation du vivant et l'identification de conceptionsobstacles sont un passage nécessaire afin d'étudier, ultérieurement, les processus d'apprentissage et l'élaboration de séquences didactiques en les considérant au profit d'une compréhension actuelle du vivant. Enfin, est présentée brièvement l'influence positive que peut avoir une formation scientifique en biologie sur la conceptualisation du vivant et sur les conceptions-obstacles, qui se voient alors décliner ${ }^{2}$.
\end{abstract}

\section{Epistemology of biology and future teachers and biologists conceptualization of living}

\begin{abstract}
The results presented in this article result from a doctoral study that was interested in the history of biology and significant philosophic conceptions that, until now, fueled the building of knowledge in biology (Simard, 2015 [1]). In this regard, a set of life conceptions was selected and circumscribed to develop a new evaluation tool considering the occurrence of one or other of conceptions among future teachers and biologists. For some of these historic conceptions, that are now considered as obsolete, the issue is that they see themselves very alive in scientific thought, even today. The characterization of the conception of living and the identification of obstacle-conceptions is a necessary step to subsequently study the learning processes and the development of teaching sequences considering them for the benefit of a current understanding of living. Finally, this study presents the positive influence of scientific training in biology on the conceptualization of the living by a decrease of the obstacle-conceptions.
\end{abstract}

\footnotetext{
${ }^{1}$ Auteure de correspondance : catherine_simard@uqar.ca

${ }^{2}$ Cette dimension de la présente étude sera davantage étayée dans le cadre d'un autre article en préparation.
} 


\section{Problématique}

Aujourd'hui, nous pouvons difficilement faire abstraction de l'histoire des savoirs, des concepts, voire des disciplines. Ils sont marqués par la dimension historique qui a canalisé le champ des possibles dans la construction même des savoirs biologiques contribuant ainsi à diverses conceptions du vivant selon les époques et qui ont chapeauté la construction des connaissances. Conséquemment, nous nous sommes demandés si l'histoire des connaissances sur le vivant, c'est-à-dire l'épistémologie de la biologie, a marqué notre conceptualisation du vivant au point d'y retrouver, encore aujourd'hui, des conceptions du vivant datant d'Aristote, de Galien ou de Linné, lesquelles pouvant être considérées comme désuètes. Et si oui, dans quelle mesure ? Il nous semble pertinent de mettre en lumière la complexité (la multidimensionnalité) de la conceptualisation du vivant chez un enseignant et/ou un biologiste. Cette étude aspire à mieux cerner les conceptions qui composent leur conceptualisation du vivant et d'en identifier les conceptions-obstacles présentes pour, ensuite, proposer des points d'appui lors d'élaboration de situations d'enseignement qui en tiendraient compte.

En milieu éducatif (école et autres), ces conceptions-obstacles peuvent être repérées dans les discours et contenus d'enseignement, dans les outils et choix didactiques ou produits de la culture scientifique qui, conséquemment, moduleront la conceptualisation du vivant chez l'apprenant (Clément, 2004 [2] ; Coquidé et Vander-Borght, 1998 [3]; Miller, Scott, Okamoto, 2006 [4]). Par ailleurs, des auteurs évoquent que les obstacles repérés auprès des apprenants peuvent, en partie, prendre leur source dans les pratiques pédagogiques teintées de ces mêmes obstacles, propres aux enseignants (Favre et Verseils, 2006 [5] ; Kochkar, 2007 [6] ; Monchamps, 1997 [7] ; Peterfalvi, 1997 [8]). Dans ce contexte, l'identification d'obstacles dans la construction des savoirs biologiques chez les enseignants et/ou biologistes ${ }^{3}$ est fondamentale (Simard, Harvey et Samson, 2013 [9]). C'est pourquoi nous avons interrogé ces principaux porteurs de cette culture scientifique en biologie au Québec, afin de caractériser leur propre conceptualisation du vivant et à savoir, s'il y a présence d'obstacles (Lesquels ? Dans quelle proportion ?). Ces derniers, acteurs et concepteurs de situations d'apprentissage, pourraient donc, à leur insu, reconduire leurs propres conceptions-obstacles dans la compréhension du vivant chez l'apprenant (Monchamps, 1997 [7]). Pour l'éviter, sous une approche réflexive, ils doivent prendre conscience de leurs propres conceptions du vivant et de celles qui représentent des obstacles afin d'être en mesure de présenter, avec recul, des situations d'apprentissage (Ibid. [7]; Mignon et Closset, 2004 [10]). Et sous l'angle de la formation, ces éléments permettront de mieux définir des séquences didactiques qui tiennent compte des divers obstacles potentiels, et ce, tant dans les objectifs et dans les stratégies didactiques pour les apprenants que lors de la formation universitaire des enseignants et biologistes (Peterfalvi, 1997 [8]; Astolfi et Peterfalvi, 1993, 1997 [11, 12]). Car, l'identification des obstacles à franchir et des conceptions à construire contribue, chez l'apprenant, à l'appropriation de modèles théoriques du vivant cohérents avec les avancées de la science contemporaine. Afin de mettre en place les bonnes conditions favorisant un enseignement centré sur les conceptions à construire et les obstacles à passer, encore faut-il que l'enseignant puisse identifier ses propres conceptions et celles représentant des obstacles. C'est donc l'une des visées de la présente étude; mettre en exergue l'ensemble des conceptions du vivant qui coexistent chez un enseignant ou biologiste. Plus précisément, la question de recherche est : Quelle est la conceptualisation du vivant d'enseignants et biologistes en formation et l'influence que peuvent avoir les connaissances en biologie sur celle-ci?

\section{Cadre théorique}

À ce point, il nous semble inévitable, dans le cadre de cette étude, d'étayer les conceptions

\footnotetext{
${ }^{3}$ Les biologistes peuvent œuvrer en milieu formel en tant que professionnels dans leur discipline, enseignants au cégep ou à l'université ou comme vulgarisateurs scientifiques en milieu non formel (dans un musée, par exemple).
} 
circonscrites dans leur contexte historique ${ }^{4}$. Documenté notamment par Atlan (1999 [13]) et Morange (2005 [14]), on remarque que ces dernières décennies ont été marquantes dans le développement des savoirs en biologie, laissant présager une rupture épistémologique imminente du paradigme du « tout génétique » pour une vision plus complexe et flexible, sous le paradigme interactionniste. Ainsi, en résonance aux savoirs biologiques actuels, la conceptualisation contemporaine du vivant fait référence à sa forme évolutive (évolutionnisme) et en reconnaissant des interrelations complexes et flexibles entre le génome et l'environnement (interactionnisme).

\section{1 Épistémologie de la biologie}

Plusieurs auteurs ont contribué à l'élaboration de ce cadre théorique, notamment Dupouey (2005 [15]), Canguilhem (1967 [16]), Atlan (1999 [13]), Pichot (1993 [17]) et Morange (2005 [14]). À la lecture de l'histoire de la biologie, on remarque que divers paradigmes ont chapeauté, selon les époques, la conceptualisation et la construction des savoirs sur le vivant. Ici, sont présentées brièvement les conceptions paradigmatiques circonscrites dans le cadre de la présente étude.

D'abord, l'animisme d'Aristote (384-322 av. J.-C.) introduit l'étude de la vie comme « la science de corps animés ». Ainsi, l'animal est pourvu d'une âme, privilège accordé à tout être vivant, l'âme étant le principe fondamental et explicatif du vivant. Le mot "âme», de racine grecque anima, signifie le souffle vital, conception qui exclut toute connotation spirituelle. Aristote prétend que le vivant est l'unité, la somme de la matière et de l'âme qui l'informe. Ainsi, l'âme n'est pas, à elle seule, le vivant qui vient se loger dans une matière inerte, mais un tout (Dupouey, 2005 [15]).

Quant à la conception finaliste, elle se définit comme le caractère de ce qui a un but et permet d'expliquer les fonctions vitales du vivant en une fin, à l'intérieur d'un projet. Le finalisme modéré d'Aristote devient sous Galien (129-200 apr. J.-C.) plus strict, du type organe-fonction, et qui va se vulgariser au point de devenir une pensée idéologique plus que philosophique. «La nature qui ne fait rien en vain d'Aristote, devient chez Galien, l'affirmation d'une providence divine particulièrement astucieuse et bienveillante (la nature ou le Créateur, selon ses propres mots) a fait l'être vivant de sorte qu'il est doté d'organes adaptés à réaliser telle ou telle fonction » (p. 131) (Pichot, 1993 [17]) .

Ces conceptions de vivant coexistent avec la conception mécaniste qui le définit comme étant soumis aux mêmes règles naturelles que la matière inerte (Canguilhem, 1967 [16]). Dans l'époque Antique, ce courant épistémologique est d'abord évoqué par les atomistes matérialistes (Leucippe et Démocrite au $\mathrm{V}^{\mathrm{e}}$ siècle av. J.-C., Épicure (341-270 av. J.-C.) et Lucrèce (94-55 av. J.-C.)). Ainsi, les atomes ne sont pas vivants; le vivant, composé d'atomes, ne peut être que dépourvu de vie. Beaucoup plus tard, l'un des points forts de ce courant autour de l'année 1628, lorsque William Harvey découvre la circulation sanguine. On parle du vivant comme étant un système de pression, de débits et de volumes. À cette même période, Descartes (1596-1650) développe le concept «d'animauxmachines " où le fonctionnement du corps et l'organisation de ses organes se comparent au mécanisme d'une horloge. Il s'oppose à tous les niveaux d'âme, vestige de l'animisme aristotélicien. Alors qu'Aristote animait tout d'une âme, Descartes mécanise le monde en faisant disparaître la notion d'une vie métaphysique (Dupouey, 2005 [15]). Cette période est donc marquée par la mécanisation de l'animal. Toutefois, Descartes ne renonce pas à l'énoncé de vie. Il considère que le cœur, organe corporel et source de chaleur, est le siège de la vie de tout être vivant. Leibniz (1714) évoquera, plus tard, le corps organique comme une sorte d'automate vivant, telle une machine dans ses moindres parties. Base des sciences modernes, il y a, dans cette conception philosophique, les

\footnotetext{
${ }^{4} A$ priori, nous tenons à mentionner que cette analyse historique, couvrant une longue période et ciblant des conceptions précises, peut perdre en subtilité sous cette forme de synthèse nécessaire pour cet article. Toutefois, mettre en valeur les traits marquants qui ont caractérisés ces conceptions circonscrites a été essentiel afin d'élaborer un questionnaire qui a mis en exergue les diverses conceptions du vivant chez les futurs enseignants et biologistes (voir Simard, 2015 [1]).
} 
prémices de la biologie moléculaire (réductionnisme) où les phénomènes biologiques sont régis par l'action mécanique de structures à découvrir. En effet, en 1962, cette conception matérialiste du vivant est bien évoquée par Kahane [18] qui mentionne, dans La vie n'existe pas !, que « la vie est le mode de raisonnement de mouvement de la matière parvenue à un degré convenable de complexité et d'organisation » (p. 228).

Tel un retour du balancier, afin de contrer cette mécanisation de la vie, la conception vitaliste, présente au cours de la deuxième moitié du $18^{\mathrm{e}}$ siècle, dominera jusqu'au début du $19^{\mathrm{e}}$ siècle (Pichot, 1993 [17]). Bichat (1771-1802) est l'un de ses représentants majeurs. Il conçoit la vie comme une matière animée par un principe vital distinct, tel un complément « d'organisation » qui, s'ajoutant à la matière, est la seule à répondre et à lutter contre les lois physico-chimiques (Atlan, 1999 [13]). Cette force organisatrice, fondement unificateur et stabilisateur, permet de résister à une destruction interne. Selon Pichot (1993 [17]), « ce n'est pas une théorie très homogène et aussi construite que l'animiste; il y a plusieurs sortes de vitalismes, mais tous s'accordent pour attribuer à l'être vivant un principe qui lutte contre les lois physiques, lesquelles sont considérées comme contraires à la vie. » (p. 525). Ainsi, l'animal composé de matière inerte prend vie par un principe particulier à la vie, immatériel (Dupouey, 2005 [15]). Bichat, sous réserve d'une mesure précise, expose une échelle de degré de vitalité selon l'enfant, l'adulte et le vieillard, de sorte que la matière ne perd jamais de ses qualités physiques, mais perd de ses qualités vitales.

Avec le développement de la chimie organique et de la thermodynamique, c'est au $19^{\mathrm{e}}$ siècle que les biologistes abandonnent l'idée qu'il existe un principe vital, voire la "vie » si elle désigne une entité cachée, divine, obscure. Par ailleurs, amorcé au siècle précédent, c'est au $18^{\mathrm{e}}$ siècle que les philosophes et scientifiques accentuent leur division. Leur indépendance sera, au $19^{\mathrm{e}}$ siècle, presque totale. De nature métaphysique et élaboré par la voie de la philosophique, le courant vitaliste décline donc sous la critique d'une science empirique et expérimentale (Pichot, 1993 [17]).

Par ailleurs, sous une approche naturaliste, tributaire d'une compréhension systématique des organismes et espèces vivantes, la conception fixiste, adoptée par Linné (1707-1778) et prolongée par Cuvier (1769-1832), correspond à la vision d'une nature qui reste immuable, où les espèces seraient apparues telles quelles au cours des temps géologiques et n'auraient subi aucune évolution depuis leur création. Linné, fils de pasteur, est près des textes sacrés. Buffon (1707-1788), va jusqu'à dire, « Dieu a créé et Linné à classé » (Picq, 2011 [19]). En effet, selon les observations de Linné, tout se passe comme s'il existait une unité qui représente un ordre de la Nature. Linné imaginait que cette classification naturelle représentait l'ordre de la Création. Entre 1809 et 1859, la conception fixiste coexiste avec la vision d'une transformation de l'espèce évoquée par Lamarck et ensuite, par Darwin (Pichot, 1993 [17]). Naturellement, sous cette conception fixiste, la conception transformiste, évoquant le mouvement et la transformation du vivant, se voit inconcevable (Dupouey, 2005 [15]). Le transformisme de Lamarck (1744-1829) est défini comme étant des modifications pendant la vie de l'animal et qui passent à la génération suivante par hérédité. Cette conception philosophique, aujourd'hui considéré comme un obstacle, a introduit les notions de temps et de transformation du vivant. Ces nouvelles perspectives aussi étonnantes que critiquées créent une rupture majeure avec les conceptions antérieures. C'est également à cette période que la biologie, comme science autonome, fait ses premiers pas. Lamarck et le naturaliste allemand Treviranus (1776-1837), proposent, de façon indépendante, le terme biologie pour désigner la science qui étudie différentes formes de vie, les conditions et lois qui régissent leur organisation et le fonctionnement des êtres vivants (Le Guyader, $2002[20])$.

Charles Darwin évoque, pour les biologistes, la naissance de la science moderne du vivant. On assiste à une rupture épistémologique certaine qui, encore aujourd'hui, demeure. La conception évolutionniste du vivant provient aussi d'une vision ordonnée de la nature : observer, comparer et classer (Picq, 2011 [19]). Cette conception n'est pas complètement incompatible avec l'époque. En revanche, dans un contexte moral où tout lien avec les animaux est impensable, cette compréhension du vivant choque. En effet, alors que l'ensemble de ses contemporains se considèrent comme des 
êtres supérieurs, spiritualisés, dans un contexte religieux fort, Darwin annonce que les hommes et les singes auraient un ancêtre commun... Cette part animale de l'être humain est difficile à accepter. Malgré les vives oppositions liées à certaines traditions morales et intellectuelles, le terrain est, malgré tout, favorable à recevoir cette nouvelle conception du vivant (Louis-Courvoisier, Baddeley, et Picq, $2014[21])$.

Néanmoins, ce n'est qu'un demi-siècle plus tard, à la lumière de la génétique et des connaissances de la présence de mutations, que le sens et la portée de l'évolutionnisme darwinien se révèlent. L'évolutionnisme, proposé initialement par Darwin (1809-1882) et rapidement enrichie par Haeckel (1834-1919) et leurs successeurs, énonce que le vivant possède une ascendance commune pour toutes les espèces (Picq, 2011 [19]). Les êtres vivants ont une histoire décrite à partir du concept de sélection naturelle (survivre et se reproduire) et comme outils, les mutations et la diversité génétique. La génétique inscrit tangiblement le potentiel adaptatif et la diversité dont une espèce est dotée et détermine comment les mutations aléatoires viennent l'enrichir sous la pression de la sélection naturelle (Dupouey, 2005 [15] ; Campbell et Reece, 2004 [22] ; Griffiths, Wessler, Lewontin, Gelbart, Suzuki et Miller, 2006 [23]). L'évolutionnisme moderne est donc la conséquence d'un processus naturel de sélection qui favorise la reproduction des organismes les mieux adaptés à un environnement donné (Bronner, 2007 [24]).

$\mathrm{Au} 20^{\mathrm{e}}$ siècle, par le développement des savoirs en biologie et des technologies, se sont succédées rapidement les conceptions réductionnisme, déterminisme et interactionnisme, tout en consolidant celle évolutionniste. Au niveau conceptuel, par les succès de la biologie moléculaire, c'est la fin d'une très longue période où la "vie » était considérée comme une propriété, donnée par un principe vital. «Rien ne nous autorise à penser qu'ils [êtres vivants] soient subordonnés à l'intervention d'un souffle, d'un principe quelconque, irréductible aux lois du monde matériel et à leur interprétation scientifique » (p. 252) (Kahane, 1962 [18]). La conception mécaniste a pris le dessus du vitalisme et, de façon définitive, dans les années 60. Entre «vivant» et «non vivant », leur unité matérielle est concédée, mais en reconnaissant une organisation particulière du vivant et son évolution. Selon Kahane, «Libre à nous de désigner sous le nom de vie, l'ensemble des manifestations particulières au degré élevé d'organisation que présentent les vivants et, dans ce sens, nous reconnaissons comme une évidence que la vie existe » (p. 253) (Ibid. [18]). Ce dernier précise que cette conception de la vie bouscule une grande partie du public et nombre de philosophes et scientifiques car elle est « en concurrence avec le poids d'une tradition lourde de métaphysique » (p. 254) (Ibid. [18]).

Par ailleurs, au cours de ces mêmes années, la recherche en biologie moléculaire et médicale a connu une révolution avec le développement de la manipulation des gènes. Dès lors, les études et les avancées sur la transformation du vivant et sur le génome ont donné un nouvel essor aux sciences biologiques et à leurs possibles applications. Ces études et leurs retombées transforment le visage des sciences biologiques contemporaines, multiplient les réflexions éthiques, sociétales et se questionnent sur les rapports entre sciences et société ainsi que sur les concepts de vie et vivant (Atlan, 1999 [13] ; Bonneuil et Thomas, 2009 [25] ; Cherlonneix, 2013 [26]).

Au départ, cette ère de la génétique s'inscrit sous la conception réductionniste, initiée par les lois de Mendel (redécouverte au début du $20^{\mathrm{e}}$ siècle) et enrichie du concept de mutation. Cette conception énonce la simplification et l'explication du vivant à travers ses gènes. Le vivant est alors défini par son bagage génétique qui, lui-même, est régi par les lois de l'hérédité. Ce bagage génétique donne une explication mécanique au développement du vivant (Campbell et Reece, 2004 [22] ; Ancet, 2004 [27]). Cette conception tente d'expliquer la vie par l'élaboration de règles héréditaires dont le support est l'ADN (Campbell et Reece, 2004 [22] ; Campbell et Mathieu, 1995 [28]). Par la suite, énoncé d'abord par Descartes, le déterminisme s'exprime sous forme de causalité : chaque effet de la réalité est produit par quelque cause initiale qu'il est possible de déterminer en remontant la chaîne des causes (Le Moigne, 1995 [29]). Au sens strict, le déterminisme génétique signifie la capacité de prédire un caractère chez le vivant à partir de son bagage génétique, la cause étant le gène et le caractère exprimé étant l'effet causé. Ce déterminisme génétique a répondu à nombre d'espoirs 
communément rencontrés dans nos sociétés. Cette conception est rassurante et sécurisante quant à la capacité de la médecine d'aujourd'hui à œuvrer à la base du vivant pour prévenir la maladie ou, du moins, l'enrayer par l'application d'une modification génétique. Cette avenue épistémologique ne considère aucunement l'effet de l'environnement sur le vivant. Pourtant, le vivant ne peut vivre dans un environnement «neutre» dans lequel ce dernier évolue depuis quelques milliards d'années, en supportant l'idée que tout n'est dirigé que par les gènes (Campbell et Reece, 2004 [22]).

Soutenue en partie par les connaissances actualisées en biologie, une rupture épistémologique semble se dessiner entre le paradigme déterministe retrouvé sous la bannière du " tout-génétique » et l'interactionnisme (Atlan, 1999 [13]). La question controversée est d'identifier dans quelle mesure le vivant est préprogrammé génétiquement ou plutôt façonné par son milieu. Cette vision plus complexe sur les notions d'interactions entre le génome et l'environnement est de plus en plus discutée par la communauté scientifique, mais pas intégré par tous les scientifiques (Clément, 2004 [2]; Clément et Forissier, 2001 [30]). Sous la conception interactionniste, le vivant est considéré comme étant l'expression de caractères résultant d'effets combinés entre sa génétique et son environnement. Cette conception tente de combler la brèche entre l'inné (ADN) et l'acquis, où l'environnement est essentiel pour qu'il y ait une activation ou inhibition des gènes (Ancet, 2004 [27]). Moteur de cette rupture épistémologique émergente, les concepts d'épigénétique et de plasticité cérébrale soutiennent cette conception du vivant (Morange, 2005 [14]).

\subsection{Obstacle épistémologique historique}

Brousseau propose une typologie qui répertorie trois types d'obstacles cognitifs : ontogénique ${ }^{5}$, didactique $^{6}$ et épistémologique (1986 [31]). Dans la conceptualisation du vivant, la présence d'une conception désuète est définie comme étant un obstacle épistémologique « lié à la fois au sujet et à la nature même de l'objet» (p. 41) (Dell'Angelo-Sauvage, 2007 [32]). De plus, dans le cadre de notre étude, l'obstacle épistémologique est aussi intimement lié au développement historique des connaissances où « la connaissance en cause a joué un rôle dans le développement historique d'un concept » qui est le « vivant» ou les différentes manifestations du vivant (p. 417) (Berthelot et Salin, 1996 [33]). Ainsi, l'obstacle épistémologique historique se définit comme étant «historiquement attesté et participe à la signification des notions auxquelles ils se rapportent. Parmi ces derniers, certains sont encore d'actualité et ne doivent pas être ignorés par l'enseignant (obstacles épistémologiques inévitables)» (p. 277) (Brousseau, 1986 [31]). Discutés avec plus de précision dans les articles de Simard, Harvey et Samson (2013, 2014 [9, 34]), cette étude a exploré un ensemble de conceptions du vivant qui sont, pour certaines d'entre elles, pointées comme obstacles épistémologiques historiques.

Dans notre recension de la documentation scientifique, les principales études répertoriées s'articulent autour de l'identification de conceptions sur le vivant et d'obstacles épistémologiques historiques pouvant entraver ou appuyer une conceptualisation contemporaine du vivant. Puisant dans l'histoire de la biologie, nombre d'auteurs se sont intéressés notamment aux conceptions déterministe et évolutionniste (Miller et al., 2006 [4] ; Atlan, 1999 [13] ; Hrairi et Coquidé, 2002 [35]). Quant aux conceptions animiste, vitaliste, finaliste et interactionniste, elles ont été marginalement traitées en contexte éducatif en France comme au Québec (Monchamp, 1997 [7] ; Mignon et Closset, 2004 [10] ; Bronner, 2007 [24]). Parmi ces conceptions, l'animisme, le vitalisme, le finalisme, le fixisme, le déterminisme strict et comportemental représentent des obstacles épistémologiques historiques et interfèrent à divers degrés dans la construction d'une conceptualisation du vivant en adéquation avec les savoirs actuels, soutenus par la communauté scientifique. Donc, reconduites dans la pensée

\footnotetext{
${ }^{5}$ L'obstacle ontogénique se réfère au développement psychogénétique de l'humain, c'est-à-dire aux modèles spontanés liés au développement «normal » d'un individu.

${ }^{6}$ L'obstacle didactique relève de conséquences artificiellement créées par des décisions didactiques, il est donc inhérent au processus d'enseignement/apprentissage.
} 
scientifique, c'est en termes d'obstacles à une conceptualisation contemporaine du vivant qu'elles sont appréhendées dans notre étude.

En effet, le finalisme et le fixisme limitent le développement adéquat des concepts relatifs à l'évolutionnisme (Bronner, 2007 [24]; Blackwell, Powell, Dukes, 2003 [36] ; Perbal, Susanne, Slachmuylder, 2006 [37]). À titre d'exemple, il apparait plus naturel d'interpréter l'évolutionnisme sous une conception finaliste. Dans un discours dit évolutionniste, les explications s'insèrent plutôt dans une logique d'intention. D'une part, la notion de " hasard» semble détenir un caractère contreintuitif. L'appropriation de cette notion exige un effort cognitif signifiant de par son niveau d'abstraction. Il devient alors plus simple de demeurer dans la pensée commune, sous une conception finaliste (Bronner, 2007 [24]). D'autre part, par la voie de la psychologie cognitive, Coley et Tanner (2012 [38]) se réfèrent plutôt à une pensée téléologique qui relève d'une prédisposition de l'humain (pensée intuitive) à réfléchir le monde sous une forme d'intentionnalité, de finalité. Cette pensée téléologique se retrouve sous les affirmations suivantes: "Les oiseaux ont des ailes parce qu'ils doivent voler » ou «Les plantes libèrent de l'oxygène, car les animaux en ont besoin pour survivre » (p. 210). L'une des explications repose sur le besoin de répondre à la question « Pourquoi? » et qui oriente les réponses vers un lien de cause à effet (Ibid. [38]). Toutefois, étendue au domaine de la biologie, cette pensée téléologique est inappropriée et fait obstacle au développement de la pensée scientifique de l'apprenant, jeune ou adulte, en altérant sa compréhension de l'évolutionnisme.

Quant aux conceptions animiste et vitaliste, elles font entrave à la compréhension des phénomènes du vivant par la façon "d'interpréter » le vivant et du statut particulier accordé à la matière vivante comparée à la matière inerte. Elles sont donc susceptibles de perturber l'accès aux concepts biologiques fondamentaux par cette difficulté à réduire les manifestations du vivant à des lois de la matière composées de différents concepts d'hérédité, biochimiques et biophysiques (Monchamp, 1997 [7]). Thouin (1998 [39]) mentionne la présence d'évocations animistes chez l'enfant pour distinguer le vivant du non-vivant et que cette conception persiste auprès de futurs enseignants et même d'enseignants en exercice [39]. Cela est également observé par Monchamp (1997 [7]) pour la conception vitaliste. C'est dans cette perspective que ce dernier a entrepris une recherche participative. Se basant sur une prise de conscience progressive de ses propres obstacles à la construction de concepts biologiques par le vitaliste, l'auteur a modifié ses stratégies d'enseignement par la mise en place de nouvelles situations d'apprentissage. Il a priorisé l'obstacle vitaliste comme point de rencontre avec l'apprenant et comme point de départ des nouveaux apprentissages par leur confrontation aux savoirs actuels. De plus, il a également transformé ses justifications théoriques en favorisant l'introduction d'outils de la physique et de la chimie (interdisciplinarité) permettant de réduire le fossé qui sépare la compréhension de la matière inerte et vivante. À la suite de cette succession de changements, il a été à même de constater une réduction de la conception vitaliste chez ses élèves.

Enfin, le déterminisme strict, retrouvé sous le paradigme du «tout génétique », constitue un obstacle à l'appropriation de concepts biologiques actuels et plus flexibles entre le bagage héréditaire et le milieu de vie, dont la plasticité cérébrale et l'épigénétique (Atlan, 1999 [13]).

$\mathrm{Au}$ regard de ces travaux, nous nous sommes interrogés à savoir : Quelles sont les conceptions existant chez les futurs enseignants et biologistes, futurs porteurs des savoirs et compétences liés à la conceptualisation et aux problématiques du vivant en milieu formel et non formel ? Retrouve-t-on, à des degrés divers, les différentes formes de conceptions sur le vivant ? Comment se structurent ces conceptions? Observe-t-on la présence de conceptions-obstacles ? Et, dans quelle proportion?

\subsection{Effet des connaissances en biologie}

Des travaux mentionnent que le fait d'expliciter une conception et de la confronter à des connaissances issues de recherches empiriques pourrait conduire à sa modification (Abrougui et 
Clément, 1996 [40]; Doudin, Pons, Martin et Lafortune 2003 [41]; Fortin, 2008 [42]). La malléabilité des conceptions sur le vivant est possible à travers l'amélioration des connaissances disciplinaires, soutenue par l'appropriation de lois ou concepts génétiques sous-jacents à l'explicitation du vivant. Ainsi, l'appropriation de connaissances en biologie semble intervenir dans la modification de conceptions du vivant, dont l'évolutionnisme, le fixisme, le vitalisme et le déterminisme génétique.

En ce qui concerne l'évolutionnisme, plus grande est la compréhension des concepts et des lois qui régissent le vivant par l'appropriation d'une culture scientifique en biologie, plus acceptée est la théorie de l'évolution (Miller et al., 2006 [4] ; Clément et Quessada, 2008 [43]). Des études révèlent également qu'une meilleure formation des enseignants par l'appropriation de concepts fondamentaux du vivant favorise la conception évolutionniste du vivant, au détriment de convictions créationnistes qui sous-entendent la conception fixiste (Ibid. [43]).

Quant à la conception vitaliste, Monchamp (1997 [7]) met en lumière l'importance d'une prise de conscience de son propre obstacle vitaliste (à titre d'enseignant en exercice) et de l'effet des connaissances sur celui-ci. Il précise que la remise en cause de sa conception vitaliste est le résultat de ses études universitaires en biochimie et en génétique et de ses lectures en épistémologie de la biologie. L'auteur témoigne également de l'impact de l'ensemble de ses connaissances sur son enseignement du vivant et, subséquemment, sur une conceptualisation plus matérialiste du vivant chez ses élèves.

Par ailleurs, Abrougui et Clément (1996 [40]) suggèrent qu'en introduisant le concept d'hérédité, suivi d'une activité pédagogique, ils sont à même de susciter un conflit cognitif significatif chez l'apprenant (10 ans), où la coexistence d'un déterminisme comportemental dit «socioculturel» et phénotypique se dissocie de façon notoire, favorisant ainsi une modification des conceptions déterministes chez l'apprenant. Kochkar (2007 [6]) a mis en évidence qu'un enseignement sur la plasticité et l'épigenèse cérébrales suscite une modification significative de la conception déterministe, mais à certaines conditions. Cette étude révèle qu'une formation préalable en biologie des apprenants va de pair avec ce changement conceptuel.

À la suite de ces constats, il est pertinent de s'interroger quant à l'influence des connaissances actualisées en biologie sur les conceptions du vivant. Les connaissances influencent-elles chacune des conceptions? Y a-t-il des conceptions qui résistent ou persistent malgré l'acquisition de connaissances relatives au vivant? Peut-on établir des liens entre la modification de certaines conceptions et l'acquisition de connaissances en biologie? Les futurs enseignants et biologistes, distincts par leur formation et leur somme de connaissances et compétences disciplinaires (biologie), présentent-ils une même conceptualisation du vivant?

\section{Méthodologie}

L'approche méthodologique quantitative a été priorisée. Sous une optique exploratoire, cette approche rend compte de l'état général du phénomène étudié. Bien que la principale limite repose sur la portée explicative des observations, elle favorise une vision globale de la situation. Ce regard général permet de mieux cerner, non seulement les relations entre l'effet des connaissances en biologie sur les conceptions du vivant, mais aussi la coexistence des conceptions et l'ampleur de leur présence.

Pour y arriver, l'étape de la construction d'un nouvel instrument de mesure adapté aux concepts ciblés a été fondamentale (processus explicité dans Simard, 2015 [1]). La collecte de données (2011) s'est réalisée par l'entremise d'une plateforme Internet de l'UQAR à laquelle les étudiants ont accès. Les étudiants dans les divers programmes d'enseignement et dans le programme de biologie constituent les sujets à l'étude. L'échantillon est formé de 359 participants ayant répondu à la totalité du questionnaire. 
Tableau 1. Nombre de répondants des études 1 et 2 selon la formation universitaire

\begin{tabular}{lc}
\hline Programme d'études universitaires & $\mathrm{N}=359$ \\
\hline Biologie & 32 \\
Éducation - préscolaire et primaire & 111 \\
Éducation - enseignement secondaire & 14 \\
Éducation - enseignement secondaire - Profil Sciences et technologie & 5 \\
Éducation - enseignement secondaire - Profil Univers social & 7 \\
Éducation - enseignement professionnel & 98 \\
Éducation - adaptation scolaire et sociale & 80 \\
Programme d'étude inconnu & 12 \\
\hline
\end{tabular}

\section{Résultats et discussion}

D'une part, cette étude aspire à mettre en lumière la complexité d'une compréhension du vivant. Elle met en exergue une conceptualisation multidimensionnelle chez les enseignants et biologistes en formation, principaux acteurs et concepteurs de situations d'apprentissage relatives au vivant dans les milieux formel et non formel. La problématique étant que leur propre conception du vivant peut ultérieurement teinter leur enseignement et reconduire cette même conceptualisation chez l'apprenant, pouvant inclure, à l'insu des deux protagonistes, des obstacles épistémologiques historiques. D'autre part, cette étude a estimé l'influence de leur culture scientifique en biologie sur leur conceptualisation du vivant. Ici, les résultats obtenus sont discutés.

\subsection{Conceptualisation du vivant chez les futurs enseignants et biologistes}

D'abord, ce qui retient notre attention et explicité davantage dans Simard et al. (2014 [34]), est que le déterministe strict et surtout le fixisme sont des conceptions rejetées par 85,5\% et 97,6\% des répondants. En contrepartie, l'évolutionnisme (accepté à 88,3\%) et l'interactionnisme (accepté à 83,0 $\%)$ sont des conceptions largement présentes dans leur conceptualisation, tel que soutenu par les savoirs actuels. En revanche, on remarque que le finalisme (accepté à 90,4\%), qui est un obstacle, prédomine sur l'évolutionnisme et l'interactionnisme dans leur conceptualisation du vivant.

Par ailleurs, l'originalité de la présente recherche est de porter un nouveau regard sur la nature multidimensionnelle des conceptions du vivant retrouvée chez un individu. Les résultats ont permis de mettre en lumière cinq grandes composantes conceptuelles du vivant coexistant chez les futurs enseignants et biologistes. Parmi celles-ci, on observe la présence de conceptions-obstacles dont le finalisme, l'animisme, le vitalisme et le déterminisme comportemental (tableau 2).

Tableau 2. Taux d'acceptation (\%) pour les cinq composantes conceptuelles du vivant

\begin{tabular}{|l|c|}
\hline Composantes & Taux d'acceptation (\%) ${ }^{\text {a }}$ \\
\hline Animisme + Vitalisme & 51,9 \\
\hline Finalisme + Évolutionnisme & 82,4 \\
\hline Interactionnisme + Évolutionnisme & 65,1 \\
\hline Déterminisme comportemental & 44,0 \\
\hline Déterminisme héréditaire & 39,1 \\
\hline $\begin{array}{l}{ }^{\mathrm{a}} \text { Constitué des répondants (étude 2, 2011) qui sont «plutôt en accord » et « en accord » avec chacun } \\
\text { des énoncés constituants une composante donnée. }\end{array}$
\end{tabular}

De cette première analyse exploratoire, on remarque qu'un répondant universitaire sur deux adhère à l'idée qu'un principe abstrait s'ajoute à la matière pour créer le vivant. Tel un paravent métaphysique, ces conceptions sont susceptibles de faire obstacle à l'interprétation même de ce qu'est 
le vivant et de ce qui distingue le vivant de la matière inerte (Monchamp, 1997 [7] ; Hrairi et Coquidé, 2002 [35]; Bachelard, 1967 [44]). Ces résultats corroborent certains éléments évoqués par Monchamp, puis Thouin, où ces conceptions se retrouvent dans la pensée enseignante (Monchamp, 1997 [7]; Thouin, 1998 [39]). En somme, les résultats de la présente étude indiquent que les conceptions animiste et vitaliste se maintiennent, non seulement chez un répondant universitaire sur deux $(51,9 \%)$, mais s'associent de façon à produire une vision métaphysique du vivant.

Cette étude a également établi un lien direct entre l'évolutionnisme et le finalisme et entre l'évolutionnisme et l'interactionnisme. Dans le premier cas de figure, l'évolutionnisme est associé tout simplement à son obstacle, le finalisme. On peut comprendre dans ce cas-ci que $82,4 \%$ des répondants envisagent l'évolutionnisme sous une perspective finaliste. Cet obstacle finaliste entrave la compréhension même des concepts relatifs à l'évolution dont l'adaptation, la sélection naturelle, les mutations et la notion de hasard. Les futurs enseignants et biologistes accorderaient, peut-être à leur insu, une forme d'intentionnalité à l'évolution du vivant.

En revanche, l'évolutionnisme s'associe également à l'interactionnisme, de sorte que $65 \%$ des répondants adhèrent aux conceptions actuelles du vivant. Néanmoins, le finalisme domine.

\subsection{Influence des connaissances en biologie sur la conceptualisation du vivant}

Afin d'estimer l'effet des connaissances sur la conceptualisation du vivant, un questionnaire sur les connaissances a permis de scinder l'ensemble des répondants en deux groupes. Selon leur performance au test de connaissances, deux groupes d'analyse ont été formés, soit Gr0 et Gr1. Le groupe Gr1 rassemble les répondants démontrant une maîtrise nettement significative des connaissances et concepts évalués. Étroitement lié aux programmes d'études en sciences, le groupe Grl (dit de « connaissance ») comprend les étudiants en biologie et ceux en enseignement secondaire en sciences et technologie. Quant au groupe Gr0, il assemble les étudiants des autres programmes d'études démontrant une certaine appropriation terminologique en génétique sans indiquer une compréhension des concepts (voir Simard, 2015 [1]). Cette classification a permis d'effectuer des analyses différenciées afin d'estimer l'effet des connaissances sur la conceptualisation du vivant. Au tableau 3 sont présentés les principaux résultats.

Tableau 3. Taux de rejet et d'acceptation (\%) des conceptions en fonction des groupes Gr0 et Gr1

\begin{tabular}{|c|c|c|c|}
\hline \multirow{2}{*}{\multicolumn{2}{|c|}{ Conceptions }} & \multicolumn{2}{|c|}{ Taux (\%) } \\
\hline & & Rejeté & Accepté \\
\hline \multirow{2}{*}{$\begin{array}{l}\text { Animisme } \\
+ \text { Vitalisme } * * *\end{array}$} & Gr0 & 44,7 & 55,3 \\
\hline & Gr1 & 80,6 & 19,4 \\
\hline \multirow{2}{*}{ Finalisme $* *$} & Gr0 & 8,4 & 91,6 \\
\hline & Gr1 & 21,0 & 79,0 \\
\hline \multirow[t]{2}{*}{$\begin{array}{l}\text { Déterminisme } \\
\text { comportemental * }\end{array}$} & Gro & 54,7 & 45,3 \\
\hline & Gr1 & 69,2 & 30,8 \\
\hline
\end{tabular}

Lorsque l'on analyse l'effet des connaissances, celles-ci ont une influence significative seulement sur les conceptions-obstacles, de sorte que le taux d'adhésion à ces explications diminue de façon à laisser place à une conceptualisation plus actuelle. Toutefois, elles ne favorisent pas davantage l'acceptation des conceptions actuelles telles que l'évolutionnisme et l'interactionnisme qui demeurent stables d'un groupe à l'autre. Cela suggère que l'appropriation de connaissances en biologie ne favorise pas une prise en charge substantielle des conceptions à construire. Ces résultats présentent plutôt un consensus ou un degré standard d'acceptation de ces deux conceptions du vivant chez tous les répondants, quelle que soit leur formation. Le contact, dès le jeune âge, avec la théorie 
de l'évolution et les divers concepts inhérents à ces conceptions du vivant peut contribuer à ces taux d'acceptation et d'explications homogènes. Jégou-Mairone (2009 [45]) mentionne que c'est un résultat typique. L'auteure énonce que la conception évolutionniste est une forme de consensus partagé chez des répondants de niveau culturel élevé où les idées évolutionnistes sont acquises. L'auteure observe un fort taux d'acceptabilité de l'idée d'évolution du vivant à laquelle $96 \%$ des enseignants du primaire y adhèrent. Néanmoins, $57 \%$ estiment manquer de connaissances pour l'enseigner.

L'influence la plus marquante a lieu sur les conceptions animiste et vitaliste, qui s'estompent de façon notoire. Cette conception, qui fait intervenir un principe vital pour donner la vie à la matière inerte, est rejetée par 80,6\% des répondants ayant une culture scientifique plus approfondie en biologie. Par contre, plus de la moitié des autres enseignants y adhèrent à 55,3\%. Face à ce résultat, on peut s'interroger sur les enseignements généraux relatifs au vivant faisant partie du parcours typique des répondants du Gr0. Il semble qu'ils soient insuffisants ou ne tiennent pas compte de cet obstacle métaphysique, de sorte qu'il se maintient notamment chez ces derniers.

Par ailleurs, la « connaissance» démontre également une influence sur le finalisme, mais dans une moindre mesure. En conséquence, les futurs enseignants en ST et les biologistes sont 12,6\% de moins à adhérer à ces explications finalistes. Malgré cela, cette conception domine dans les explications du vivant, quel que soit le groupe. Ainsi, en dépit de l'enseignement reçu, les résultats indiquent que le parcours académique n'a pas permis de rompre avec l'obstacle finaliste chez une large majorité de futurs enseignants et biologistes. Force est de constater que cet obstacle se maintient jusqu'au niveau universitaire et peut vraisemblablement se prolonger dans des pratiques enseignantes.

En somme, les connaissances en biologie, par l'entremise de la formation, ont une influence sur la conceptualisation du vivant, de sorte qu'elles favorisent une conceptualisation plus actuelle du vivant par la diminution de la présence de conceptions-obstacles, mais sans les atténuer totalement.

\section{Conclusion}

Cette étude porte un nouveau regard quant à la nature multidimensionnelle des conceptions du vivant. Cinq grandes composantes conceptuelles ont été mises en lumière. Ainsi, des explications de nature animiste, vitaliste, évolutionniste, finaliste, interactionniste, déterministe héréditaire et déterministe comportemental ont émergé et coexistent de façon significative chez les futurs enseignants et biologistes. Ces derniers, à titre d'apprenants universitaires et porteurs des savoirs en biologie en milieu éducatif, ont une conceptualisation du vivant marquée par la coexistence de diverses conceptions, incluant des conceptions-obstacles.

Ce n'est pas étonnant, qu'aujourd'hui encore, de grandes conceptions retrouvées dans l'histoire de la biologie coexistent toujours, malgré l'état désuet de certaines par rapport aux savoirs scientifiques contemporains. Ce qui surprend toutefois, c'est le taux d'adhérence aux explications qui sont sous la coupe de conceptions-obstacles. Une forte majorité des répondants explique le vivant par l'entremise d'une pensée finaliste... Un enseignant sur deux (Gr0) explique le vivant par un principe vital, abstrait... Dans un contexte où les sciences et la technologie sont largement encouragées et investissent nos institutions scolaires, à titre de culture scientifique, on peut s'interroger sur ce qui est véhiculé et/ou compris par les porteurs de cette culture en regard des savoirs actuels relatifs au vivant. Cette étude souhaite donc proposer des pistes de réflexions à cet égard.

En somme, d'une part, l'étude a mis en perspective la présence d'une compréhension interactionniste-évolutionniste du vivant chez une majorité des enseignants et biologistes, correspondant à une conceptualisation contemporaine du vivant. Cependant, la formation scientifique (biologistes par rapport aux enseignants) ne semble pas influencer davantage cette compréhension actuelle du vivant. D'autre part, l'étude a également mis en lumière l'étendue de l'adhésion aux explications finaliste-évolutionniste du vivant, de même qu'elle a révélé la présence et l'association 
des conceptions animiste et vitaliste. Enfin, l'étude témoigne de la présence et de la dissociation des conceptions déterministe héréditaire et déterministe comportemental chez les répondants. Face aux conceptions-obstacles, la formation scientifique a eu un effet positif quant à leur modification, mais à divers degrés. L'influence la plus marquante se constate au niveau des conceptions animiste et vitaliste qui s'estompent de façon significative. Toutefois, le finalisme a éprouvé de légères modifications car cette conception se retrouve toujours chez une majorité d'entre eux.

L'importance à accorder à ces conceptions-obstacles, aux conceptions à construire et à une culture scientifique plus développée dans le cadre de la formation initiale et/ou continue des enseignants et auprès des biologistes est majeure pour une conceptualisation contemporaine du vivant. À cet égard, cette mise en valeur de profils conceptuels contribuera aux réflexions et stratégies didactiques lors de l'élaboration de situations didactiques au profit d'une compréhension actuelle du vivant.

\section{Références}

1. C. Simard, Conceptions paradigmatiques du vivant et attitudes envers les enjeux relatifs au vivant : Influence des connaissances en biologie d'enseignants et biologistes en formation, Thèse de doctorat inédite, Université du Québec à Rimouski, Canada (2015).

2. P. Clément, Science et idéologie : exemples en didactique et épistémologie de la biologie, Actes du colloque Sciences, Médias et Société, ENS-LSH (2004).

3. M. Coquidé, C. Vander Borght, Des recherches en didactique de la biologie: finalités, problématiques, concepts et productions (1988-1998), Aster, 27, 95-123 (1998).

4. J. D. Miller, E.C. Scott, S. Okamoto, Public acceptance of evolution, Science, 313, 765-766 (2006).

5. D. Favre, I. Verseils, Étude de l'acquisition et du réinvestissement du concept de surface portante, Aster, 25, 33-57 (1997).

6. M. Kochkar, Les déterminismes biologiques. Analyse des conceptions et des changements conceptuels consécutifs à un enseignement sur l'épigenèse cérébrale chez des enseignants et des apprenants tunisiens, Thèse de doctorat inédite, Université Lyon 1 et ISEFC, Université de Tunis (2007).

7. A. Monchamp, Quand les obstacles ouvrent des perspectives pédagogiques : Récit d'un itinéraire personnel, Aster, 25, 59-91 (1997).

8. B. Peterfalvi, Enseignants et élèves face aux obstacles, Aster, 25, 3-7 (1997).

9. C. Simard, L. Harvey, G. Samson, Épistémologie spécifique à la biologie : rapports aux savoirs disciplinaires et perspective d'obstacles à la compréhension du vivant, Esprit Critique, Revue internationale de sociologie et de sciences sociales, 17, 110-122 (2013).

10. J. Mignon, J.-L. Closset, Recherches en didactique de la biologie consacrée à l'évolution biologique, Probio-Revue, 4, 217-231 (2004).

11. J.-P. Astolfi, B. Peterfalvi, Obstacles et construction de situations didactiques en sciences expérimentales, Aster, 16, 103-141 (1993).

12. J.-P. Astolfi, B. Peterfalvi, Stratégies de travail des obstacles : Dispositifs et ressorts, Aster, 25, 194-216 (1997).

13. H. Atlan, La fin du tout génétique? Nouveaux paradigmes en biologie, (INRA Editions, Paris, 1999).

14. M. Morange, L'épigénétique: un domaine de recherche aux multiples facettes, Médecine sciences, 21, 4, 339 (2005).

15. P. Dupouey, Épistémologie de la biologie : La connaissance du vivant, (Armand Colin, Paris, 2005).

16. G. Canguilhem, La connaissance de la vie (Vrin, Paris (1967).

17. A. Pichot, Histoire de la notion de vie (Éditions Gallimard, Paris, 1993).

18. E. Kahane, La vie n'existe pas, (Éditions Rationalistes, Paris, 1962).

19. P. Picq, Voir autrement l'humain, Communication dans le cadre de l'Espace pour la vie, 
Montréal (2011).

20. H. Le Guyader, La notion d'évolution, Communication à l'Université de tous les savoirs, Canal $U(2002)$.

21. M. Louis-Courvoisier, M. Baddeley, P. Picq, Mon grand-père était-il un grand singe ? Communication dans le cadre du cycle «Histoire vivante», Maison de l'histoire, Université de Genève (2014).

22. N. A., Campbell, B. Reece, Biologie (Éditions du Renouveau Pédagogique, Québec, 2004).

23. A. J. F. Griffiths, S. R. Wessler, R. C. Lewontin, W. M. Gelbart, D. T. Suzuki, J. H. Miller, Introduction à l'analyse génétique (De Boeck, Bruxelles, 2006).

24. G. Bronner, La résistance au darwinisme: croyances et raisonnements, Revue française de sociologie, 48, 3, 587-607 (2007).

25. C. Bonneuil, F. Thomas, Gènes, pouvoirs et profits: Recherche publique et régimes de production des savoirs de Mendel aux OGM (Versailles, Édition Quae, 2009).

26. L. Cherlonneix, Nouvelles représentations de la vie en biologie et philosophie du vivant. La sculpture du vivant à l'échelle de l'interdisciplinarité (De Boeck, Bruxelles, 2013).

27. P. Ancet, Le déterminisme génétique et la liberté de choix, Communication présentée au Colloque génomique-génoéthique et anthropologique, Université de Montréal, Montréal (2004).

28. N. A. Campbell, R. Mathieu, Biologie, (De Boeck Université, Bruxelles, 1995).

29. J. L. Le Moigne, Les épistémologies constructivistes (Presses universitaires de France, Paris, 1995).

30. P. Clément, T. Forissier, L'identité biologique n'est pas uniquement génétique : un défi pour un enseignement citoyen, Conférence de LIRDHIST, Université Claude Bernard Lyon 1 (2001).

31. G. Brousseau, Obstacles épistémologiques, conflits socio-cognitifs et ingénierie didactique, in N. Bednarz et C. Garnier, Obstacles épistémologique, conflits socio-cognitifs et ingénierie didactique, (p. 277-285) (CIRADE, les éditions Agence d'Arc inc., Montréal 1986).

32. M. Dell'Angelo-Sauvage, De l'école au collège, le rapport au vivant d'élèves de 10 à 12 ans : En quoi les enseignements en SVT en $\sigma^{e}$ font-ils évoluer le rapport au vivant des élèves? Thèse de doctorat, ENS-Cachan, France (2007).

33. R. Berthelot, M.-H. Salin. L'enseignement des angles aux élèves de 10 à 13 ans : Identification d'un obstacle didactique, Revue des sciences de l'éducation, 22, 2, 417-442 (1996).

34. C. Simard, L. Harvey, G. Samson, Regard multidimensionnel des conceptions du vivant; situation en contexte québécois, RDST, 9, 79-102 (2014).

35. S. Hrairi, M. Coquidé, Attitudes d'élèves tunisiens par rapport à l'évolution biologique, Aster, 35, 149-163 (2002).

36. W. Blackwell, M. Powell, G. Dukes, The problem of student acceptance of evolution, Journal of Biological Education, 37, 2, 58-67 (2003).

37. L. Perbal, C. Susanne, J.L. Slachmuylder, Évaluation de l'opinion des étudiants de l'enseignement secondaire et supérieur de Bruxelles vis-à-vis des concepts d'évolution (humaine), Antropo, 12, 1-26 (2006).

38. J. Coley, K. Tanner, Common origins of diverse misconceptions: Cognitive principles and the development of biology thinking. CBE-Life Science Education, 11, 3, 209-215 (2012).

39. M. Thouin, Que peuvent nous apprendre les conceptions en sciences de la nature? Québec français, 110, 48-50 (1998).

40. M. Abrougui, P. Clément, Évolution des conceptions d'élèves de 10 ans sur la génétique à la suite d'activités scolaires incluant une visite scolaire à la Cité des enfants, Didaskalia, 8, 33-60 (1996).

41. P.-A. Doudin, C. Pons, D. Martin, L. Lafortune, Conceptions et connaissances : analyse de deux types de rapports au savoir, in L. Lafortune, C. Deaudelin, P.-A. Doudin et D. Martin (dir.), Conceptions, croyances et représentations en math, sciences et technos (p. 7-24) (Presses de l'Université du Québec, Québec, 2003).

42. C. Fortin, L'enseignement de l'évolution au lycée, entre description et explication, in M. Coquidé et S. Tirard (dir.), L'évolution du vivant : Un enseignement à risque? (p. 15-34) (Vuilbert, Paris, 
2008).

43. P. Clément, M.-P. Quessada, Les convictions créationnistes et/ou évolutionnistes d'enseignants en biologie : une étude comparative dans dix-neuf pays, Natures Sciences Sociétés, 16, 2, 154158 (2008).

44. G. Bachelard, La formation de l'esprit scientifique (Vrin, Paris, 1967).

45. C. Jégou-Mairone, L'enseignement de l'évolution des espèces vivantes à l'école primaire française. Rapport au savoir d'enseignants et d'élèves de cycle 3, Thèse de doctorat inédite, Université d'Aix Marseille I, Université de Provence (2009). 